\title{
Abnormal expression of miR-133a in patients with acute myocardial infarction following radical surgery for gastric cancer and the underlying mechanism
}

\author{
JING YU, XUFEN CAO, YE ZHENG, LIQIU YAN and JIAWANG WANG \\ Department of Cardiology, Cangzhou Central Hospital of Hebei, Cangzhou, Hebei 061000, P.R. China
}

Received December 24, 2016; Accepted September 6, 2017

DOI: $10.3892 / \mathrm{mmr} .2018 .9541$

\begin{abstract}
The present study aimed to investigate the expression of microRNA (miR)-133a in patients with or without acute myocardial infarction (AMI) following radical surgery for gastric cancer, and to explore its underlying mechanisms. Blood samples were collected from patients with or without AMI in order to detect the expression levels of miR-133a and endothelial injury markers. In addition, an AMI rat model was established. Reverse transcription-quantitative polymerase chain reaction was used to detect the mRNA expression levels of miR-133a and B-cell lymphoma 2-like 1 (Bcl211). In addition, an ELISA assay was used for endothelial injury marker analysis. To investigate the effects of miR-133a on human umbilical vein endothelial cells (HUVECs), a miR-133a inhibitor was used. Cell proliferation and apoptosis were subsequently detected using an MTT assay and flow cytometry. Western blot analysis was also conducted to detect $\mathrm{Bcl} 211$ protein expression. The results suggested that patients with AMI exhibited significantly increased expression of endothelial injury markers (von Willebrand factor, heart-type fatty acid-binding protein and cardiac troponin I) and miR-133a in blood samples compared with patients without AMI. In addition, treatment with a miR-133a mimic was able to upregulate the expression of endothelial injury markers in an AMI rat model, whereas treatment with a miR-133a inhibitor had the opposite effect. Furthermore, cellular experiments indicated that a miR-133a inhibitor could promote HUVEC proliferation and reduce cell apoptosis. The present results also confirmed that miR-133a directly targets $\mathrm{Bcl} 211$ and negatively regulates $\mathrm{Bcl} 211$ expression. In conclusion, the results of the present study suggested that miR-133a was involved in the endothelial injury process after AMI by targeting Bcl211.
\end{abstract}

Correspondence to: Dr Jing Yu, Department of Cardiology, Cangzhou Central Hospital of Hebei, 16 Xinhua Road, Cangzhou, Hebei 061000, P.R. China

E-mail: yujingdrcz@163.com

Key words: acute myocardial infarction, gastric cancer, microRNA-133a, endothelial injury, cardiac markers

\section{Introduction}

Gastric cancer (GC) is the fifth most common cancer worldwide and is considered a serious threat to human health, which negatively affects quality of life. The main treatment options for GC include surgery, chemotherapy and radiation therapy; however, each treatment is associated with complications. Surgery is the most common method of GC treatment and is a radical type of treatment: The primary tumor is resected along with the metastatic lymph nodes and the infiltrated tissue, with no tumor remaining (1). Cardiovascular complications are common in elderly patients with GC during the perioperative period and are the main cause of mortality in elderly patients following surgery. Acute myocardial infarction (AMI) is the most serious cardiovascular complication that threatens patient survival.

AMI, which is a serious cardiovascular disease (2), is characterized by inflammation, cardiomyocyte apoptosis and cardiac fibrosis (3). AMI is a primary disease that threatens human health due to its sudden onset, rapid progression and high mortality rate $(4,5)$. It has previously been confirmed that there are no obvious clinical features in the early stages of AMI (6). AMI can result in left ventricular dilatation and heart failure, which can eventually lead to mortality (7). Therefore, the early discovery, diagnosis and treatment of this disease are important for the prognosis of patients with AMI.

MicroRNAs (miRNAs/miRs) are conserved, small, non-coding RNA molecules that negatively regulate the expression of target genes (8). Abnormal miRNA expression has been demonstrated in various diseases, particularly in cancer $(9,10)$. Numerous studies have indicated that miRNAs participate in the occurrence and progression of cardiovascular diseases, including AMI (11-16). Liu et al reported that miR-150 serves a cardioprotective role in AMI via regulating monocyte cell migration and proinflammatory cytokine production (17). In addition, miR-92 is highly expressed in patients with AMI, and is involved in the endothelial injury process following AMI, whereas miR-92 inhibition may protect endothelial cells after AMI (18).

Previous studies have suggested that miR-133a expression is reduced in patients with GC (19), whereas it is highly expressed in patients with AMI (20) compared with in healthy controls. However, the expression of miR-133a in patients with or without AMI following radical surgery for GC remains unclear. The present study aimed to investigate the expression levels of 
miR-133a in patients with or without AMI following radical surgery for GC, and to explore its underlying mechanisms.

\section{Materials and methods}

Clinical samples. The present study was approved by the Human Ethics Committee Review Board at Cangzhou Central Hospital of Hebei (Cangzhou, China), and informed consent was obtained from all patients. A total of 20 blood samples were obtained from patients (age range, 46-74 years; sex ratio, 1:1) that were diagnosed with AMI 3 days after undergoing radical surgery for GC at Cangzhou Central Hospital of Hebei between February 2015 and December 2016. In addition, 20 blood samples were obtained from patients (age range, 44-71 years; sex ratio, 1:1) that did not suffer from AMI 3 days after undergoing radical surgery for GC. No patients had received any radiotherapy or chemotherapy prior to surgery. Blood samples were used to detect miRNA-133a and endothelial injury marker expression.

AMI rat model (21). The rat study was approved by the Ethics Committee at Cangzhou Central Hospital of Hebei. A total of 20 male Wistar rats weighing 180-220 g were purchased from the Vital River Company (Beijing, China) and bred in standard conditions (temperature, $21 \pm 1^{\circ} \mathrm{C}$; humidity, 55-60\%). They were given water and food ad libitum. All the rats were anesthetized by intraperitoneal administration of pentobarbital $(50 \mathrm{mg} / \mathrm{kg})$, and then AMI rat models were established by ligating the left anterior descending coronary artery (LAD), as previously described (21). The control rats underwent a sham operation.

A miR-133a mimic (sense, 5'UUUGGUCCCCUUCAA CCAGCUG3' and antisense, 5'GCUGGUUGAAGGGGA CCAAAUU3'); miR-133a inhibitor (5'CAGCUGGUUGAA GGGGACCAAA3') and negative control (NC; sense, 5'UUC UCCGAACGUGUCACGUTT3' and antisense, 5'ACGUGA CACGUUCGGAGAATT3') all obtained from Shanghai Pharmaceutical Technology Co., Ltd.; $2 \mu \mathrm{g}$ mimic, inhibitor or NC in a total of $120 \mu \mathrm{l}$ DMEM were injected into three areas of the myocardium of anterior left ventricular wall near the LAD during AMI surgery. The rats were then divided into four groups (18): Control group, in which 5 rats that underwent the sham operation were injected with a vector; model group, in which 5 rats in the AMI model group were injected with NC; miR-133a mimic group, in which 5 rats in the AMI model group were injected with a miR-133a mimic; miR-133a inhibitor group, in which 5 rats in the AMI model group were injected with a miR-133a inhibitor. All rats were sacrificed 3 days following LAD ligation. Then blood was collected from the retroorbital plexus and immediately flash-frozen in liquid nitrogen and stored at $-80^{\circ} \mathrm{C}$.

ELISA. To analyze the endothelial injury markers, including cardiac troponin I (cTnI; cat. no. Ab200016; Abcam, Cambridge, UK), heart-type fatty acid-binding protein (H-FABP; cat. no. CSB-E09185h; Cusabio Biotech Co., Ltd., College Park, MD, USA) and von Willebrand factor (vWF; cat. no. Ab108918; Abcam), blood samples from patients and rats were collected. Subsequently, ELISA analyses were performed according to the manufacturer's protocols.
Cell culture and transfection. Human umbilical vein endothelial cells (HUVECs) and the 293T cell line were obtained from the American Type Culture Collection (Manassas, VA, USA). HUVECs were cultured in endothelial growth medium (Gibco; Thermo Fisher Scientific, Inc., Waltham, MA, USA) in a humidified atmosphere containing $5 \% \mathrm{CO}_{2}$ at $37^{\circ} \mathrm{C}$. The 293 T cells were grown in high glucose Dulbecco's modified Eagle's medium (Gibco; Thermo Fisher Scientific, Inc.) medium containing 10\% fetal bovine serum (FBS; Gibco; Thermo Fisher Scientific, Inc.) at $5 \% \mathrm{CO}_{2}$ and $37^{\circ} \mathrm{C}$.

A miR-133a inhibitor (5'CAGCUGGUUGAAGGGGAC CAAA3'; $100 \mathrm{nM}$ ), NC (sense, 5'UUCUCCGAACGUGUC ACGUTT3' and antisense, 5'ACGUGACACGUUCGGAGA ATT3'; $50 \mathrm{nM}$ ) or $2 \mu \mathrm{l}$ B-cell lymphoma 2 (Bcl-2)-like 1 (Bcl211) small interfering (si)RNA (cat. no. sc-43630; Santa Cruz Biotechnology, Inc., Dallas, TX, USA) were transfected into HUVECs $\left(5 \times 10^{4}\right.$ cells/well) using the Lipofectamine ${ }^{\circledR}$ LTX kit (Invitrogen; Thermo Fisher Scientific, Inc.) according to the manufacturer's protocol. The HUVECs were divided into four groups: The control group, in which cells were not transfected; the NC group, in which cells were transfected with $\mathrm{NC}$; the inhibitor group, in which cells were transfected with a miR-133a inhibitor; and the inhibitor + Bcl211 siRNA group, in which cells were cotransfected with a miR-133a inhibitor and Bcl211 siRNA. Pre-experimental results demonstrated that Blc211 siRNA alone significantly decreased Bcl211 expression. Thus, the present study did not examine cells transfected with the Bcl211 siRNA alone.

Luciferase activity assay. To verify whether miR-133a directly targets the $3^{\prime}$ untranslated region (3'UTR) of Bcl211, Bcl211-3'UTR-wild type (WT) and Bcl211-3'UTR-mutant (MUT) vectors, containing wild type and mutated 3'UTR of Bcl211 mRNA respectively, were established as previously described (22). $293 \mathrm{~T}$ cells were seeded in a 24 -well plate $\left(5 \times 10^{4}\right.$ cells/well) and were then co-transfected with Bcl211-3'UTR-WT or Bcl211-3'UTR-MUT and 50 nM miR-133a or $100 \mathrm{nM}$ NC vector using Lipofectamine ${ }^{\circledR} 2000$ transfection reagent (Invitrogen; Thermo Fisher Scientific, Inc.) according to the manufacturer's protocol. Following transfection for $48 \mathrm{~h}$, Luciferase activity was analyzed using a Dual-Luciferase Reporter Assay kit (Promega Corporation, Madison, WI, USA) according to the manufacturer's protocol.

Cell proliferation and apoptosis assays. A total of $48 \mathrm{~h}$ post-transfection, an MTT assay was applied to evaluate cell proliferation. Cells $\left(3 \times 10^{3}\right.$ cells per well) were seeded in a 96-well plate, and were incubated for $48 \mathrm{~h}$ followed by staining with $20 \mu \mathrm{l}$ MTT $(5 \mathrm{~g} / \mathrm{l})$ at $37^{\circ} \mathrm{C}$ for $4 \mathrm{~h}$. Subsequently, the supernatant was discarded and the precipitate was dissolved following the addition of $200 \mu \mathrm{l}$ dimethyl sulfoxide. The optical density value of each sample was measured at $490 \mathrm{~nm}$ using a spectrophotometer. Experiments were performed in triplicate. Cell apoptosis was determined using an Annexin V-fluorescein isothiocyanate (FITC)/propidium iodide (PI) Apoptosis Detection kit (BD Biosciences, Franklin Lakes, NJ, USA) using flow cytometry. In brief, $48 \mathrm{~h}$ following cell transfection, cells were rinsed with cold PBS. Cells $\left(5 \times 10^{5}\right.$ cells/well) were then labeled with Annexin V-FITC and propidium iodide, according to the manufacturer's protocol. Flow cytometry 
(BD Biosciences, Franklin Lakes. NJ, USA) was used for cell apoptosis analysis. WinMDI version 2.5 (Purdue University Cytometry Laboratories; www.cyto.purdue.edu/flowcyt/software/Catalog.htm) was used for data analysis and each test was repeated in triplicate.

Reverse transcription-quantitative polymerase chain reaction $(R T-q P C R)$. Total RNA was extracted from blood or cells using TRIzol reagent (Invitrogen; Thermo Fisher Scientific, Inc.) according to the manufacturer's protocol. GAPDH or U6 were used as internal controls. The PrimeScript RT reagent kit (Takara Bio, Inc., Otsu, Japan) was used to transcribe total RNA into cDNA according to the manufacturer's protocol. Amplification conditions for RT-PCR were as follows: $16^{\circ} \mathrm{C}$ for $30 \mathrm{~min}, 42^{\circ} \mathrm{C}$ for $30 \mathrm{~min}, 85^{\circ} \mathrm{C}$ for $5 \mathrm{~min}$ and holding at $4^{\circ} \mathrm{C}$. qPCR analysis was conducted using SYBR Premix Ex Taq (Takara, Bio, Inc.). qPCR was conducted with following conditions: $95^{\circ} \mathrm{C}$ for $10 \mathrm{~min}$, followed by 37 cycles at $95^{\circ} \mathrm{C}$ for $15 \mathrm{sec}$ and $72^{\circ} \mathrm{C}$ for $30 \mathrm{sec}$. Relative gene expression was calculated using the $2^{-\Delta \Delta \mathrm{Cq}}$ method (23). Each experiment was performed in triplicate. The PCR primer sequences are listed in Table I.

Western blot analysis. Cells were harvested and lysed using radioimmunoprecipitation assay butter (Cell Signaling Technology, Inc., Danvers, MA, USA). A bicinchoninic acid protein assay kit (Beyotime Institute of Biotechnology, Haimen, China) was used for protein quantification. Protein samples $(25 \mu \mathrm{g} /$ lane $)$ were separated by $12 \%$ SDS-PAGE and were then transferred to a polyvinylidene fluoride membrane. The membranes were blocked in Tris-buffered saline with $0.1 \%$ Tween $^{\circledR}$ (TBST) with $5 \%$ skimmed milk for $1.5 \mathrm{~h}$ at room temperature. Subsequently, the membranes were incubated with primary antibodies against Bcl2l1 (cat. no. 2764; 1:1,000; Cell Signaling Technology, Inc.) and GAPDH (cat. no. 8884; 1:2,000; Cell Signaling Technology, Inc.) at $4^{\circ} \mathrm{C}$ overnight. After washing with TBST, the membranes were incubated with anti-rabbit IgG, HRP-linked Antibody (cat. no. 7074; 1:5,000; Cell Signaling Technology, Inc.) at room temperature for $4 \mathrm{~h}$. Protein bands were visualized using enhanced chemiluminescence substrates (EMD Millipore, Billerica, MA, USA) and images were then captured.

Statistical analysis. SPSS 18.0 statistical software (SPSS, Inc., Chicago, IL, USA) was used to conduct all statistical analyses. Each experiment was repeated in triplicate. Data are presented as the mean \pm standard deviation. Comparisons between two groups were performed using Student's t-test. Comparisons between multiple groups were performed using one-way analysis of variance followed by Tukey's post hoc test. $\mathrm{P}<0.05$ was considered to indicate a statistically significant difference.

\section{Results}

Patients with AMI exhibit higher miR-133a and endothelial injury marker expression. The expression levels of miR-133a and endothelial injury markers were detected in patients with or without AMI following radical surgery for GC using RT-qPCR and ELISA, respectively. The results demonstrated that compared with the control patients, patients with AMI exhibited higher miR-133a and endothelial injury marker expression (Fig. 1).
Table I. Primer sequences for quantitative polymerase chain reaction.

\begin{tabular}{ll}
\hline Gene & \multicolumn{1}{c}{ Sequence (5'-3') } \\
\hline Bcl211-F & GGTGGTTGACTTTCTCTCCT \\
Bcl211-R & GCATCTCCTTGTCTACGCTT \\
GAPDH-F & CTTTGGTATCGTGGAAGGACTC \\
GAPDH-R & GTAGAGGCAGGGATGATGTTCT \\
miR-133a-F & TTTGGTCCCCTTCAACCAGCTG \\
miR-133a-R & TAAACCAAGGTAAAATGGTCGA \\
U6-F & CTCGCTTCGGCAGCACA \\
U6-R & AACGCTTCACGAATTTGCGT
\end{tabular}

Bcl211, B-cell lymphoma 2-like 1; miR-133a, microRNA-133a; F, forward; R, reverse.

miR-133a regulates endothelial injury marker expression. The present study also investigated the effects of miR-133a on endothelial injury marker expression in rats, following the establishment of an AMI rat model (21). As presented in Fig. 2, miR-133a expression was significantly increased in the AMI rat model; treatment with a miR-133a mimic further enhanced the expression of miR-133a, whereas miR-133a expression was decreased in the miR-133a inhibitor group. Compared with the control group, the expression levels of the endothelial injury markers (vWF, H-FABP and cTnI) were significantly increased in the AMI rat model. In addition, treatment with the miR-133a mimic enhanced the expression levels of endothelial injury markers (vWF, H-FABP and cTnI) in the AMI rat model, whereas the miR-133a inhibitor had the opposite effect.

miR-133a directly targets Bcl2ll and regulates Bcl2l1 expression. To investigate the mechanisms underlying the effects of miR-133a on the regulation of endothelial injury, the target genes of miR-133a were investigated using TargetScan (http://www.targetscan.org/vert_71/) and miRanda (http://www.microrna.org/microrna/home.do) databases. Numerous target genes were discovered, including Bcl211. As Bcl211 serves critical roles in cell proliferation and apoptosis, Bcl211 was selected for further analysis. Subsequently, a luciferase activity assay was used to confirm the predictions. The results indicated that miR-133a directly targets Bcl211 (Fig. 3).

The present study also confirmed that miR-133a negatively regulated Bcl211 expression in HUVECs. Transfection with the miR-133a inhibitor significantly increased Bcl211 expression; however, this increase was reduced following transfection with the Bcl2l1 siRNA (Fig. 4).

Effects of miR-133a on HUVEC cell proliferation. To explore the underlying mechanisms of miR-133a in the regulation of endothelial injury, the present study determined the effects of miR-133a on HUVEC cell proliferation using an MTT assay. The results suggested that transfection with the miR-133a inhibitor significantly increased HUVEC cell proliferation, whereas this increase was abrogated by Bcl211 siRNA (Fig. 5). 

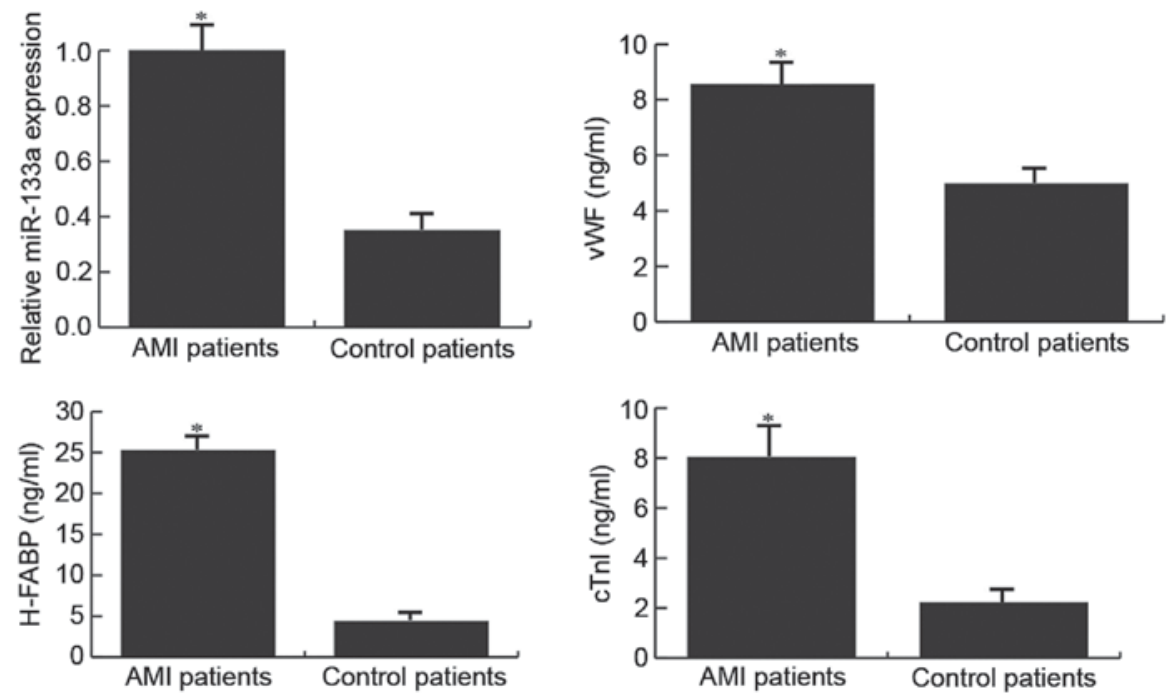

Figure 1. Expression levels of miR-133a and endothelial injury markers (vWF, H-FABP and cTnI) in patients with or without AMI. The expression levels of miR-133a, and vWF, H-FABP and cTnI, were detected in patients using reverse transcription-quantitative polymerase chain reaction and ELISA, respectively. AMI patients, patients with AMI following radical surgery for gastric cancer; Control patients, patients without AMI following radical surgery for gastric cancer. Data are presented as the mean \pm standard deviation. " $\mathrm{P}<0.05$ vs. the Control patients. AMI, acute myocardial infarction; cTnI, cardiac troponin I; H-FABP, heart-type fatty acid-binding protein; miR-133a, microRNA-133a; vWF, Von Willebrand factor.

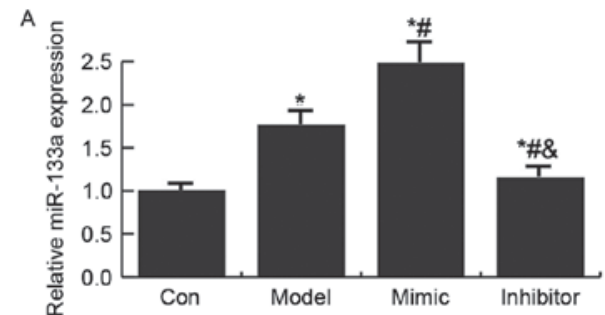

C

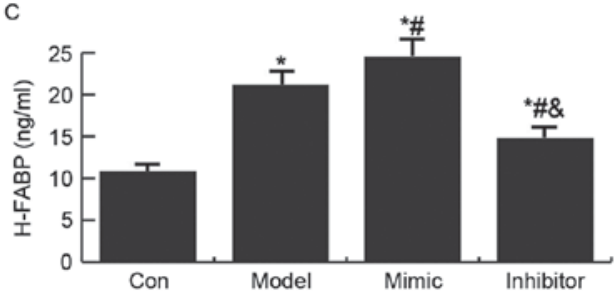

B

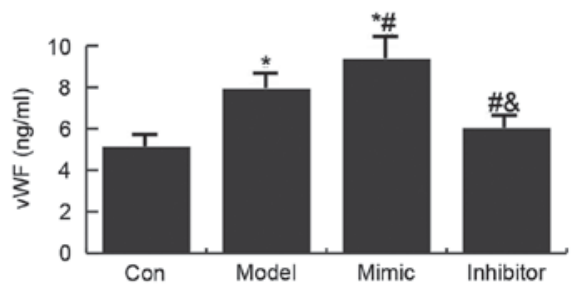

D

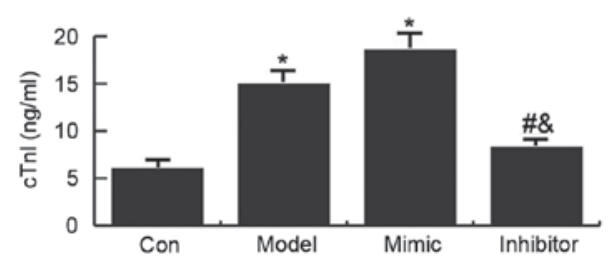

Figure 2. Expression levels of miR-133a and endothelial injury markers (vWF, H-FABP and cTnI) in a rat model of acute myocardial infarction. (A) Quantification of the expression levels of miR-133a in model rats, as determined by RT-qPCR. Expression levels of (B) vWF, (C) H-FABP and (D) cTnI in model rats, as determined by ELISA. Data are presented as the mean \pm standard deviation. ${ }^{*} \mathrm{P}<0.05$ vs. the Con group; ${ }^{*} \mathrm{P}<0.05$ vs. the Model group; ${ }^{\star} \mathrm{P}<0.05$ vs. the Mimic group. Con: Control group, rats that underwent the sham operation were injected with a vector; Model: Model group, rats in the AMI model group were injected with NC; mimic, miR-133a mimic group, rats in the AMI model group were injected with a miR-133a mimic; inhibitor, miR-133a inhibitor group, rats in the AMI model group were injected with a miR-133a inhibitor; cTnI, cardiac troponin I; H-FABP, heart-type fatty acid-binding protein; miR-133a, microRNA-133a; vWF, Von Willebrand factor.

Effects of miR-133a on HUVEC apoptosis. Finally, an Annexin V-FITC/PI Apoptosis Detection kit was used to analyze the effects of miR-133a on apoptosis of HUVECs. As presented in Fig. 6, compared with in the control and NC groups, the apoptotic rate of HUVECs was reduced in the miR-133a inhibitor group, whereas transfection with Bcl211 siRNA was able to reverse the miR-133a inhibitor-induced reduction in cell apoptosis.

\section{Discussion}

Taken together, the present study demonstrated that compared with the control patients, patients with AMI following radical surgery for GC exhibited higher miR-133a and endothelial injury marker expression. In addition, miR-133a could promote the expression of endothelial injury markers (vWF, H-FABP and $\mathrm{cTnI}$ ), whereas miR-133a inhibition could promote HUVEC proliferation and reduce cell apoptosis by targeting $\mathrm{Bcl} 211$. These results indicated that miR-133a was associated with endothelial injury following AMI by targeting Bcl211.

With the extensive application of electrocardiograms, perioperative cardiovascular complications during surgery for gastrointestinal tumors have garnered attention. However, the causes of perioperative cardiovascular complications of gastrointestinal tumors are very complex. Elderly patients often have the pathological basis of atherosclerosis, which increases the 


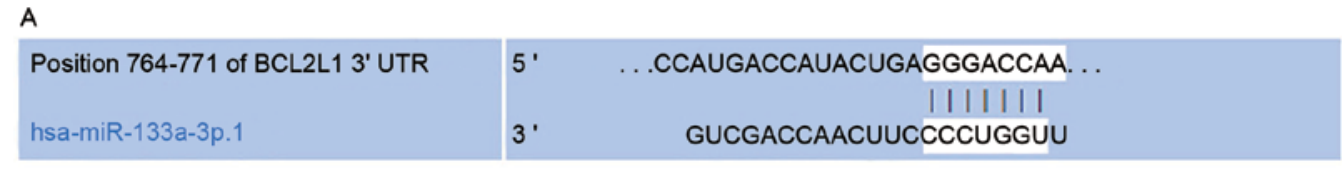

B
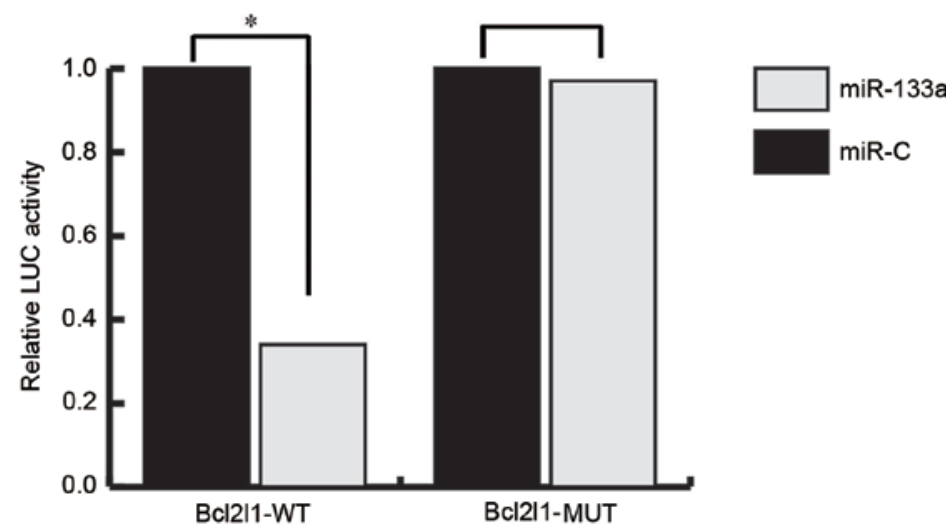

Figure 3. Bcl211 is a direct target of miR-133a. (A) Interaction between miR-133a and the 3'UTR of Bcl211 was predicted using TargetScan. (B) LUC activity of a reporter containing Bcl211-WT 3'UTR or Bcl211-MUT 3'. Bcl211-MUT indicates the Bcl211 3'UTR with a mutation in the miR-133a binding site. All data are presented as the mean \pm standard deviation of three independent experiments. ${ }^{*} \mathrm{P}<0.05$ vs. the control group. Bcl211, B-cell lymphoma 2-like 1; LUC, luciferase; miR-133a, microRNA-133a; MUT, mutant; UTR, untranslated region; WT, wild type.
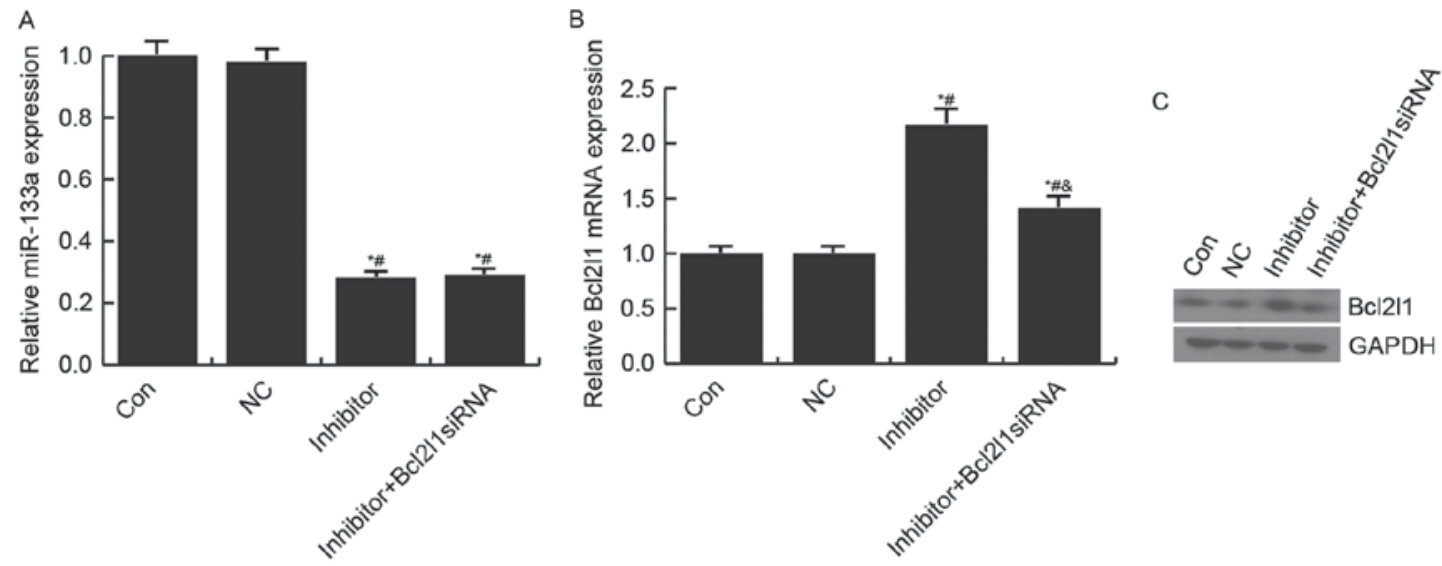

Figure 4. miR-133a negatively regulates Bcl211 expression. A total of $48 \mathrm{~h}$ post-transfection, miR-133a and Bcl211 expression were detected using reverse transcription-quantitative polymerase chain reaction and western blot analysis. (A) miR-133a expression; (B) Bcl211 mRNA expression; (C) Bcl211 protein expression. Con, untreated HUVECs; NC: HUVECs transfected with NC; inhibitor, HUVECs transfected with a miR-133a inhibitor; inhibitor + Bcl211 siRNA: HUVECs cotransfected with a miR-133a inhibitor and Bcll12 siRNA. Data are presented as the mean \pm standard deviation. "P<0.05 vs. the Con group; ${ }^{\text {"P }} \mathrm{P}<0.05$ vs. the NC group; ${ }^{\&} \mathrm{P}<0.05$ vs. the inhibitor group. Bcl211, B-cell lymphoma 2-like 1; HUVECs, human umbilical vein endothelial cells; miR-133a, microRNA-133a; NC, negative control; siRNA, small interfering RNA.

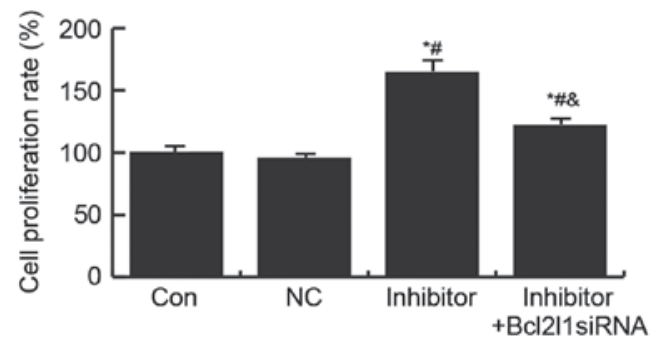

Figure 5. Effects of miR-133a on HUVEC proliferation. A total of $48 \mathrm{~h}$ post-transfection, HUVEC proliferation was determined by MTT assay. Con, untreated HUVECs; NC: HUVECs transfected with NC; inhibitor, HUVECs transfected with a miR-133a inhibitor; inhibitor + Bcl211 siRNA, HUVECs cotransfected with a miR-133a inhibitor and Bcl112 siRNA. Data are presented as the mean \pm standard deviation. ${ }^{*} \mathrm{P}<0.05$ vs. the Con group; ${ }^{~} \mathrm{P}<0.05$ vs. the NC group; ${ }^{\circledR} \mathrm{P}<0.05$ vs. the inhibitor group. Bcl211, B-cell lymphoma 2-like 1; HUVECs, human umbilical vein endothelial cells; miR-133a, microRNA-133a; $\mathrm{NC}$, negative control; siRNA, small interfering RNA. risk of AMI (24). AMI seriously threatens human survival, particularly in patients with AMI following tumor surgery; therefore, it is necessary to explore AMI pathogenesis, and to research novel diagnostic markers and treatment methods.

miRNAs have been reported to be associated with cardiovascular diseases, due to their involvement in the regulation of a wide range of processes, including gene expression control (25). Numerous studies have reported that miRNAs participate in endothelial injury $(26,27)$. Although a previous study has demonstrated that miR-133a expression is increased in the plasma of patients with AMI (20), the mechanism remained to be elucidated. The present study detected the upregulation of miR-133a and endothelial injury markers (vWF, H-FABP and $\mathrm{cTnI}$ ) in patients with AMI. To elucidate the functional roles of miR-133a in AMI, a rat model of AMI was generated. The results demonstrated that miR-133a was able to promote 

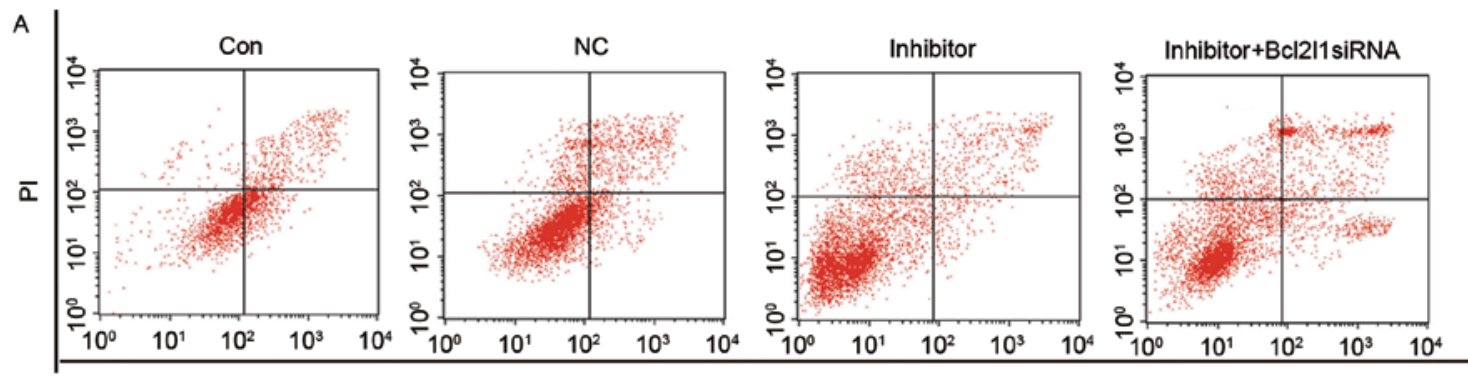

FITC

B

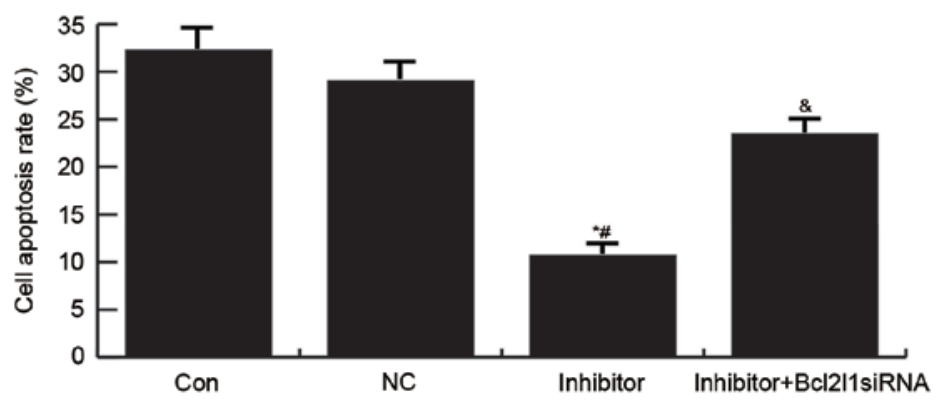

Figure 6. Effects of miR-133a on HUVEC apoptosis. (A) A total of $48 \mathrm{~h}$ post-transfection, HUVEC apoptosis was determined by flow cytometry. Con, untreated HUVECs; NC: HUVECs transfected with NC; inhibitor, HUVECs transfected with a miR-133a inhibitor; inhibitor + Bcl211 siRNA, HUVECs cotransfected with a miR-133a inhibitor and Bcl112 siRNA. (B) Data are presented as the mean \pm standard deviation. " $\mathrm{P}<0.05$ vs. the Con group; $\mathrm{P}<0.05$ vs. the NC group; \& $\mathrm{P}<0.05$ vs. the inhibitor group. Bcl211, B-cell lymphoma 2-like 1; FITC, fluorescein isothiocyanate; HUVECs, human umbilical vein endothelial cells; miR-133a, microRNA-133a; NC, negative control; PI, propidium iodide; siRNA, small interfering RNA.

the expression of endothelial injury markers (vWF, H-FABP and $\mathrm{cTnI}$ ) in a rat model of AMI, whereas treatment with the miR-133a inhibitor had the opposite effect. These data indicated that miR-133a may participate in endothelial injury.

The original indication of improvement following MI is endothelial activation, determined by the enhanced cell proliferation ability and reduced apoptosis (28). To further investigate the underlying mechanisms of miR-133a in the regulation of endothelial injury in AMI, Bcl211 was initially identified as a direct target gene of miR-133a. The protein encoded by the $\mathrm{Bcl} 211$ gene belongs to the $\mathrm{Bcl}-2$ protein family and functions as an anti-apoptotic molecule. The present study subsequently determined the effects of miR-133a on HUVEC cell proliferation and apoptosis. The results demonstrated that compared with in the control and NC groups, transfection with a miR-133a inhibitor was able to promote HUVEC proliferation and reduce cell apoptosis by upregulating $\mathrm{Bcl} 211$ gene expression.

In conclusion, the present study is the first, to the best of the authors' knowledge, to demonstrate that patients with AMI following radical surgery for GC had higher miR-133a expression compared with patients without AMI. In addition, the results indicated that miR-133a could regulate the endothelial injury process following AMI though the modulation of endothelial cells via targeting Bcl211. Therefore, miR-133a may be considered a novel therapeutic target in the future treatment of AMI; however, whether miR-133a may be targeted as a preventative intervention for AMI following radical surgery for $\mathrm{GC}$ requires further investigation.

\section{Acknowledgements}

Not applicable.

\section{Funding}

No funding was received.

\section{Availability of data and materials}

The analyzed data sets generated during the present study are available from the corresponding author on reasonable request.

\section{Authors' contributions}

JY collaborated in the design of the present study. JY, XC and YZ were responsible for data accession and analysis. JY, XC, YZ, LY and JW collaborated to interpret results and develop the manuscript.

\section{Ethics approval and consent to participate}

The present study was approved by the Human Ethics Committee Review Board at Cangzhou Central Hospital of Hebei (Cangzhou, China), and informed consent was obtained from all patients.

\section{Consent for publication}

Not applicable.

\section{Competing interests}

The authors declare that they have no competing interests.

\section{References}

1. Shi Y and Zhou Y: The role of surgery in the treatment of gastric cancer. J Surg Oncol 101: 687-692, 2010. 
2. White HD and Chew DP: Acute myocardial infarction. Lancet 372: 570-584, 2008.

3. Bogomolov AN, Kozlov KL, Kurochkina ON and Olesiuk IB: Coronary stenting in elderly patients with acute myocardial infarction (review). Adv Gerontol 26: 151-160, 2013 (In Russian).

4. Lipinski MJ, Escárcega RO, D'Ascenzo F, Magalhães MA Baker NC, Torguson R, Chen F, Epstein SE, Miró O, Llorens P, et al: A systematic review and collaborative metaanalysis to determine the incremental value of copeptin for rapid rule-out of acute myocardial infarction. Am J Cardiol 113: 1581-1591, 2014.

5. Velibey Y, Erbay A, Ozkurt E, Usta E and Akin F: Acute myocardial infarction associated with blood transfusion: Case report and literature review. Transfus Apher Sci 50: 260-262, 2014.

6. Yahalom M, Roguin N, Suleiman K and Turgeman Y: Clinical signifcance of conditions presenting with ECG changes mimicking acute myocardial infarction. Int J Angiol 22: 115-122, 2013.

7. Mozarian D, Benjamin EJ, Go AS, Arnett DK, Blaha MJ, Cushman M, de Ferranti S, Després JP, Fullerton HJ, Howard VJ, et al: Heart disease and stroke statistics update: A report from the American Heart Association. Circulation 131: e29-e322, 2015.

8. Bartel DP: MicroRNAs: Target recognition and regulatory functions. Cell 136: 215-233, 2009.

9. Yang Z, Han Y, Cheng K, Zhang G and Wang X: miR-99a directly targets the mTOR signalling pathway in breast cancer side population cells. Cell Prolif 47: 587-595, 2014.

10. Niu G, Li B, Sun J and Sun L: miR-454 is down-regulated in osteosarcomas and suppresses cell proliferation and invasion by directly targeting c-Met. Cell Prolif 48: 348-355, 2015.

11. Sala V, Bergerone S, Gatti S, Gallo S, Ponzetto A, Ponzetto C and Crepaldi T: MicroRNAs in myocardial ischemia: Identifying new targets and tools for treating heart disease. New frontiers for miR-medicine. Cell Mol Life Sci 71: 1439-1452, 2014.

12. Thum T, Gross C, Fiedler J, Fischer T, Kissler S, Bussen M, Galuppo P, Just S, Rottbauer W and Frantz S: MicroRNA-21 contributes to myocardial disease by stimulating MAP kinase signalling in fbroblasts. Nature 456: 980-984, 2008.

13. Wang X, Zhang X, Ren XP, Chen J, Liu H, Yang J, Medvedovic M, Hu Z and Fan GC: MicroRNA-494 targeting both proapoptotic and antiapoptotic proteins protects against ischemia/reperfusion-induced cardiac injury. Circulation 122: 1308-1318, 2010.

14. Fiedler J, Jazbutyte V, Kirchmaier BC, Gupta SK, Lorenzen J, Hartmann D, Galuppo P, Kneitz S, Pena JT, Sohn-Lee C, et al: MicroRNA-24 regulates vascularity after myocardial infarction. Circulation 124: 720-730, 2011.

15. Hinkel R, Penzkofer D, Zühlke S, Fischer A, Husada W, Xu QF, Baloch E, van Rooij E, Zeiher AM, Kupatt C and Dimmeler S: Inhibition of microRNA-92a protects against ischemia/reperfusion injury in a large-animal model. Circulation 128: 1066-1075, 2013
16. Devaux Y, Vausort M, McCann GP,Zangrando J, Kelly D, Razvi N, Zhang L, Ng LL, Wagner DR and Squire IB: MicroRNA-150: A novel marker of left ventricular remodeling after acute myocardial infarction. Circ Cardiovasc Genet 6: 290-298, 2013.

17. Liu Z, Ye P, Wang S, Wu J, Sun Y, Zhang A, Ren L, Cheng C, Huang X, Wang K, et al: MicroRNA-150 protects the heart from injury by inhibiting monocyte accumulation in a mouse model of acute myocardial infarction. Circ Cardiovasc Genet 8: 11-20, 2015.

18. Liu H, Li G, Zhao W and Hu Y: Inhibition of MiR-92a may protect endothelial cells after acute myocardial infarction in rats: Role of KLF2/4. Med Sci Monit 22: 2451-2462, 2016.

19. Gong Y, Ren J, Liu K and Tang LM: Tumor suppressor role miR-133a in gastric cancer by repressing IGF1R. World J Gastroenterol 21: 2949-2958, 2015.

20. Wang F, Long G, Zhao C, Li H, Chaugai S, Wang Y, Chen C and Wang DW: Plasma microRNA-133a is a new marker for both acute myocardial infarction and underlying coronary artery stenosis. J Transl Med 11: 222, 2013

21. Zhang D, Zhu L, Li C, Mu J, Fu Y, Zhu Q, Zhou Z, Liu P and Han C: Sialyltransferase7A, a Klf4-responsive gene, promotes cardiomyocyte apoptosis during myocardial infarction. Basic Res Cardiol 110: 28, 2015.

22. Zhang GJ, Zhou H, Xiao HX, Li Y and Zhou T: Up-regulation of miR-224 promotes cancer cell proliferation and invasion and predicts relapse of colorectal cancer. Cancer Cell Int 13: 104, 2013.

23. Livak KJ and Schmittgen TD: Analysis of relative gene expression data using real-time quantitative PCR and the 2(-Delta Delta C(T)) method. Methods 25: 402-408, 2001.

24. Agarwal S, Sud K, Thakkar B, Menon V, Jaber WA and Kapadia SR: Changing trends of atherosclerotic risk factors among patients with acute myocardial infarction and acute ischemic stroke. Am J Cardiol 119: 1532-1541, 2017.

25. Small EM and Olson EN: Pervasive roles of microRNAs in cardiovascular biology. Nature 469: 336-342, 2011

26. Bonauer A, Carmona G, Iwasaki M, Mione M, Koyanagi M, Fischer A, Burchfield J, Fox H, Doebele C, Ohtani K, et al: MicroRNA-92a controls angiogenesis and functional recovery of ischemic tissues in mice. Science 324: 1710-1713, 2009.

27. Zhou J, Wang KC, Wu W, Subramaniam S, Shyy JY, Chiu JJ, Li JY and Chien S: MicroRNA-21 targets peroxisome proliferators-activated receptor-alpha in an autoregulatory loop to modulate flow-induced endothelial inflammation. Proc Natl Acad Sci USA 108: 10355-10360, 2011.

28. Tedgui A and Mallat Z: Cytokines in atherosclerosis: Pathogenic and regulatory pathways. Physiol Rev 86: 515-581, 2006.

c) () $\Theta$ This work is licensed under a Creative Commons Attribution-NonCommercial-NoDerivatives 4.0 International (CC BY-NC-ND 4.0) License. 